\title{
PERFORMANCE ASSESSMENT OF THE OVERSET GRID METHOD FOR NUMERICAL WAVE TANK EXPERIMENTS IN THE OPENFOAM ENVIRONMENT
}

\author{
Christian Windt* \\ Centre for Ocean Energy Research \\ Maynooth University \\ Co. Kildare, Ireland \\ Email: christian.windt.2017@mumail.ie
}

\author{
Benazzou Akram \\ Department for Energy and Environmental Systems \\ Ecole des mines de Nantes \\ Nantes, France
}

\author{
Josh Davidson \\ Centre for Ocean Energy Research \\ Maynooth University \\ Co. Kildare, Ireland \\ John V. Ringwood \\ Centre for Ocean Energy Research \\ Maynooth University \\ Co. Kildare, Ireland
}

\begin{abstract}
To maximise the energy output of wave energy converters (WECs), large structural motions are desired. When simulating WEC performance in Computational Fluid Dynamics (CFD) based numerical wave tanks, these motions must be explicitly accommodated in the computational domain. Using well established mesh morphing (MM) methods, this explicit accommodation results in deformation of control volumes $(\mathrm{CV}) /$ mesh. Thus, large amplitude WEC oscillations may lead to highly distorted CVs and push MM models beyond the limits of numerical stability. While advanced numerical mesh motion methods, such as overset grids, have been developed in commercial CFD codes to overcome these issues, little use of these methods can be found in WEC analysis. However, recently the overset grid method (OSG) has been made available to a wider user community through its release in the open source CFD environment OpenFOAM [1,2]. To evaluate the performance of the OSG, this paper will compare the classical MM method and the OSG against experimental tank test data of the WaveStar device [3].
\end{abstract}

\footnotetext{
*Address all correspondence to this author.
}

\section{INTRODUCTION}

Industry and academia have progressed on many ideas and techniques to harvest ocean wave energy resources [4]. In order to design a cost competitive and durable WEC, engineers rely on experimental and numerical tools [5]. Within the latter category, an increased interest in high-fidelity non-linear numerical modelling by the means of CFD can be observed [6].

Avoiding linearisation, as applied in widely used lowfidelity models, CFD-based numerical wave tanks (CNWTs) are able to capture all relevant hydrodynamic non-linearities. The gain in fidelity, however, comes at increased computational cost compared to linearised models [7]. One reason for the additional cost is the complex, explicit accommodation of structural motion in the computational domain by the means of dynamic mesh motion methods (DyMMMs).

Besides increasing the computational cost, DyMMMs can introduce numerical stability issues, hence modelling limitations, when large structural displacements are considered. By virtue of the nature of WEC systems, i.e. resonant motion in several degrees of freedom for maximum energy capture [8], these limits are of particular concern for CNWT WEC experiments. 
One of the most widely employed approaches to incorporate significant structural motion in the CNWT are body fitted approaches. Considering either fixed grid connectivity or nonfixed connectivity, these methods require considerable effort in order to ensure sufficient grid quality throughout the spatial domain for the duration of the simulation $[9,10]$. In the case of fixed grid connectivity, body motion can only be accounted for by mesh morphing, where the displacement is diffused within the domain. While this procedure can be stated as computationally efficient, numerical errors are potentially introduced due to poor grid quality (i.e. high aspect ratios and highly skewed cells). Hence, mostly small, single degree of freedom (DOF) motions are considered when employing this method. Examples considering MM applied to different WEC systems can be found in a number of publications, e.g. [8,11-14].

If grid connectivity does not need to be retained, re-meshing can be applied. Compared to MM, re-meshing allows for larger body displacements but, in turn, significantly increases the computational overhead and potentially introduces numerical interpolation errors into the solution. Examples of a re-meshing strategy for CNWT WEC experiments can be found in [15-17].

For the specific case of single rotational DOFs, another procedure can be identified. Using so-called arbitrary mesh interfaces (AMIs), sliding interfaces between grid blocks are employed, allowing for large rotation angles without distorting or re-meshing the computational domain. Problems, however, can occur during interpolation of the solution over the grid interfaces. A well-known example of this methodology is the numerical model of the flap type oscillating wave surge converter, Oyster, described in $[18,19]$.

More recently, advanced approaches for the solution of the dynamic mesh motion problem have been developed, i.e. the Cartesian Cut-Cell method [9], the fast-fictitious-domain method [20], the Fractional Area-Volume Obstacle Representation (FAVOR) technique [21], or the OSG [22]. In the latter, also referred to as the Chimera method, two grids are internally static with fixed grid connectivity, but are able to move relative to each other. Interpolation between grids is then needed to transfer solutions across grid interfaces. Compared to body- fitted approaches, this method increases the numerical stability when considering large structural motions; however, OSG potentially increases the computational cost and may suffer from numerical interpolation errors. The OSG has been successfully applied in the fields of aerodynamic engineering (e.g. [23-25]) or classical naval architecture (e.g. [26-28]). Nevertheless, only a small number of CNWT WEC experiments have been performed, all of which were implemented in the commercial CFD solver STAR-CCM+ [29-35]. The relatively limited usage of the OSG in the wave energy field can be reasoned as follows:

1. the extensively larger computational cost [36]

2. limited availability of the algorithm in CFD software
3. introduction of numerical errors in a volume of fluid (VOF) environment, such as violation of mass conservation [37]

Recently, the limitation in 2. has been overcome with the OSG being made freely available to a wider user community, through the code release in the open-source CFD toolbox OpenFOAM, in two independent forks of the software documented in [1] and [2]. By overcoming many of the limitations of classical DyMMMs, the OSG is a very interesting option, which could possibly be of benefit for CNWT WEC experiments. To assess the potential value of OSG for CNWT experiments, this paper will compare its performance against the traditional fixed connectivity, boundary fitted MM method.

To evaluate the performance of the OSG, this paper will compare the classical MM method and the OSG against experimental tank test data from free decay tests performed on the WaveStar WEC device [3].

The remainder of the paper is organised as follows. Sec. 2 provides a more detailed description of the two DyMMMs considered in this study. Sec. 3 then describes the experimental tank tests used for the validation study. Sec. 4 includes the description of the setup of the CNWT. Here, we will distinguish between the solver (cf. Sec. 4.1) and the grid settings (cf. Sec. 4.2). Furthermore, due to the differences in nature of the tested methods, the subsections are split, covering the MM and OSG methods independently. Sec. 5 then presents the results of the validation study for the MM (cf. Sec. 5.1) and the OSG method (cf. Sec. 5.2). Finally, conclusions are drawn in Sec. 6.

\section{DYNAMIC MESH MOTION}

This section will introduce the two DyMMMs compared in this paper. For further insight, the interested reader is referred to the presented references.

\subsection{Mesh Morphing}

In a Finite Volume Method (FVM) algorithm, if grid connectivity should be retained (meaning no topological changes) in the grid, MM is the classical method to accommodate body motion in the computationl domain. As depicted in Fig. 1, the displacement of the body (boundary) is diffused into the domain by solving the Laplacian equation:

$$
\nabla \cdot(k \nabla \mathbf{u})=0
$$

where $k$ describes the diffusivity and $\mathbf{u}$ the velocity of the moving boundary [38]. The displacement of the body (boundary) leads to a deformation of single CVs, while the total volume of all CVs is kept constant through the simulation. Depending on the implementation, the diffusivity factor, $k$, gives control over the 
grid quality during mesh deformation. In the OpenFOAM environment, distance-based diffusivity is employed, where the user specifies an inner and outer distance (cf. Fig. 1); between these distances mesh deformation is allowed and prohibited elsewhere.

The deformation of the original good quality mesh can lead to poor grid quality, such as large aspect ratios and/or highly skewed cells, resulting in numerical stability issues. The reduction in mesh quality depends on the layout of the numerical domain, the choice of the inner and outer distances, and the amplitude of the structural motion. If the amplitude of the body motion is (roughly) known a priori, the simulation can be set up such that the likelyhood of numerical instability from mesh distortion is reduced. However, if the dynamics are not know a priori, time consuming preliminary studies must be performed.

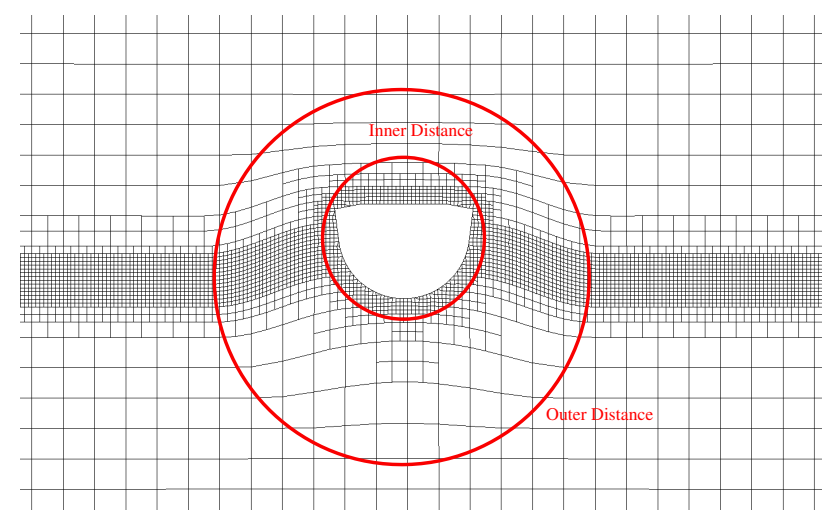

FIGURE 1: Illustration of the MM method. In the area between inner and outer distance mesh deformation is allowed

\subsection{Overset Grids}

In the OSG, (at least) two grids (background and bodyfitted) are defined, which may arbitrarily overlay each other (cf. Fig. 2). The different grids are internally static, thereby retaining their original structure and quality, but are allowed to move relative to each other. In order to pass information between the different grids, interpolation has to be performed. This interpolation can lead to conservation and convergence issues and represents the biggest challenge of this method [37].

The OSG can be split into the four sequential steps:

1. identification of hole cells

2. identification of fringe cells

3. identification of donor cells

4. interpolation between fringe and donor cells

For dynamic simulation all steps have to be performed at every time step. Hole cells embrace cells in the background grid, lying inside the moving body. These cells are marked and blanked out during the solution process (cf. Fig. 2b). This procedure is the main cause for the extensive computational cost of the OSG [39]. In the next step, cells adjacent to hole cells are identified as fringe cells. Likewise, cell at the outer boundary of the body-fitted grid are also identified as fringe cells (cf. Fig. 2c). These cells are used as boundary cells in the solution procedure. Boundary values for fringe cells are determined through solution interpolation. In the third step, the interpolation partners on both grids, the donor cells, are identified [40,41]. Lastly, interpolation between fringe and donor cells is performed.

The quality of the numerical results is directly affected by the four steps explained above and hence is related to the employed interpolation scheme and the problem discretisation in the background and body-fitted grid.

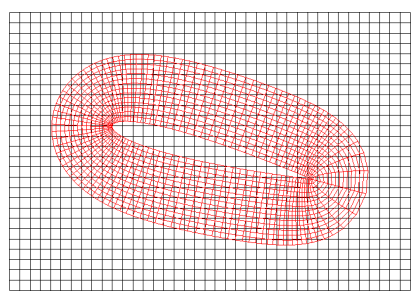

(a) Background (black) and body-fitted grid (red)

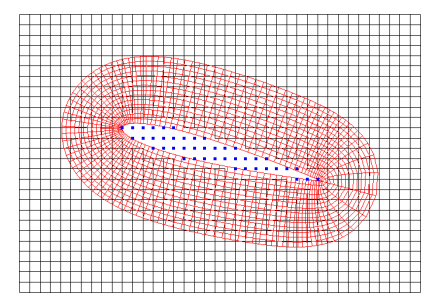

(b) Identification of hole cells: Hole points marked blue

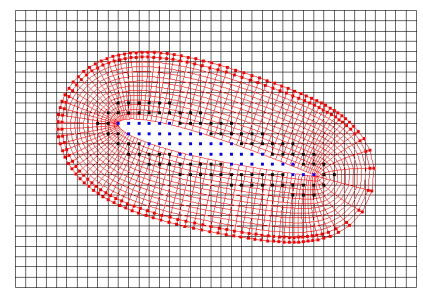

(c) Identification of fringe cells on the background grid (marked black) and the body-fitted grid (marked red)

FIGURE 2: Illustration of the OSG steps: Identification of hole (b) and fringe cells (c). The identification of donor cells is not shown above. The black grid represents the background grid, the red grid represents the body-fitted grid. (Fig. adopted from [40])

\section{EXPERIMENTAL SETUP}

In this study, experimental tank test data will be used to validate the numerical solution. This validation allows physical checking of the two different DyMMMs and defines the correct solution. The experimental results used in this study are based on free decay tests of a 1:10 scale single DOF, pitching WEC; the WaveStar device (cf. Fig. 3). The experiments were conducted at the Ocean Wave Basin at Plymouth University and are documented in [3]. 

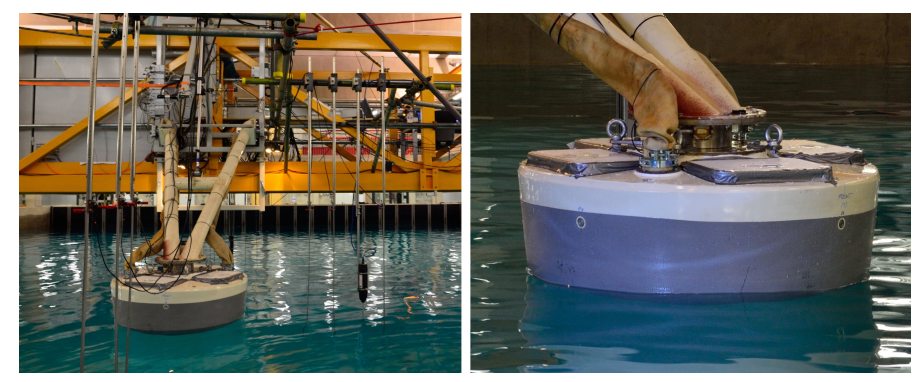

FIGURE 3: 1:10 scale WaveStar device in Plymouth Ocean Wave basin (Fig. adopted from [3])

In the free decay tests, the float is initially displaced from its equilibrium position and then released, resulting in a decayed oscillation around its equilibrium. The decaying body motion is experimentally measured via piston displacement of the hydraulic cylinder (cf. Fig. 4). Through geometric considerations, the heave position of a reference point on the float can be extracted. In the following, float heave position will be compared to numerical results. For validation, two different tests with two different initial inclinations $\alpha$ of the floating system are considered, hereafter referred to as FD1 and FD2. The initial inclination is $\alpha=-8.8^{\circ}$ and $\alpha=-5.2^{\circ}$ for FD1 and FD2, respectively.

System properties (mass, inertia, etc.) are listed in Tab. 1. A schematic of the experimental test setup, including significant dimensions, is depicted in Fig. 4.

TABLE 1: SYSTEM PROPERTIES OF 1:10 WAVESTAR MODEL TAKEN FROM $[3,13]$

Mass (Float \& Arm)

$220 \mathrm{~kg}$

Inertia

$124 \mathrm{~kg} \mathrm{~m}^{2}$

Centre of Mass (CoM) of the floating system in

equilibrium relative to the hinge position:

$\mathrm{X}$

$\mathrm{y}$

Z

$1.3954 \mathrm{~m}$

$0.0 \mathrm{~m}$

$-1.3305 \mathrm{~m}$

Submergence (in equilibrium)

$0.4 \mathrm{~m}$

Water depth $d$

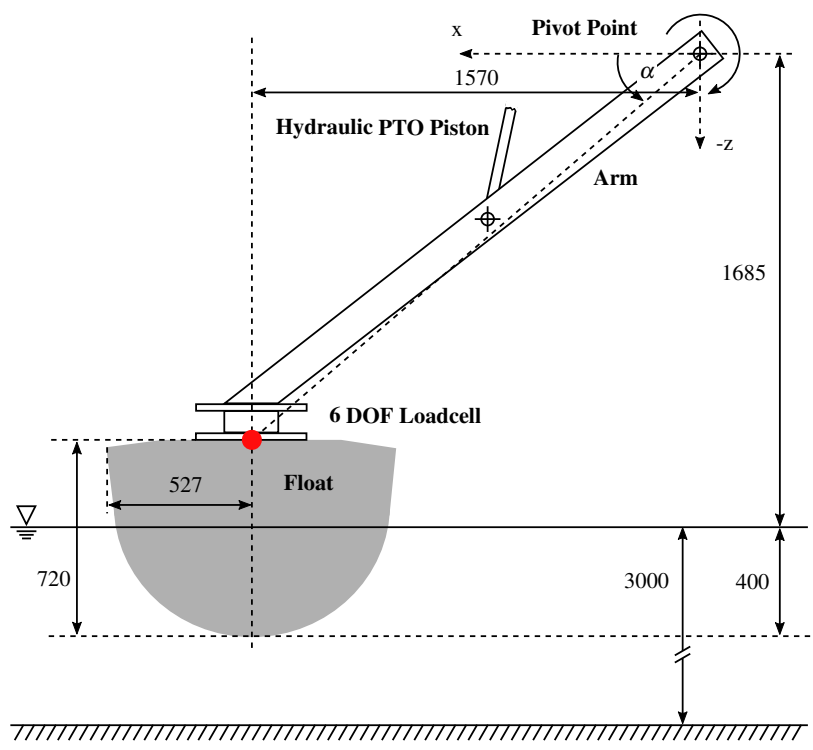

FIGURE 4: Schematic of the experimental setup including the main dimensions (in $\mathrm{mm}$ ). The reference point is marked in red. Schematic not to scale. (Fig. adopted from $[3,13]$ )

\section{NUMERICAL SETUP}

For this paper, all simulations are performed using the opensource CFD toolbox OpenFOAM [42]. More specifically, the ESI fork, version v1706 [1], including the newly implemented OSG, was employed. All simulations are run on a dedicated server using Intel Xeon(R) E5-2446 CPUs with 2.40Ghz.

In OpenFOAM, the Navier-Stokes equations (2) and (3) are solved using the FVM. To account for the two phase flow, the Volume of Fluid (VoF) method is used.

$$
\nabla \cdot \mathbf{u}=0
$$

$$
\frac{\partial(\rho \mathbf{u})}{\partial t}+\nabla \cdot(\rho \mathbf{u u})=-\nabla p+\nabla \cdot \mathbf{T}+\rho \mathbf{f}_{b}+\mathbf{s} \rho \mathbf{U}
$$

In Eqn. (2) and (3) $t$ represents time, $\mathbf{u}$ the fluid velocity, $p$ the fluid pressure, $\rho$ the fluid density, $\mathbf{T}$ the stress tensor and $\mathbf{f}_{b}$ external forces such as gravity. The last term on the right hand side of Eqn. (3), $\mathbf{s} \rho \mathbf{U}$ describes a dissipation term used to implement an efficient numerical beach, where the variable field $\mathbf{s}$ controls the strength of the dissipation, equaling zero at the start of the beach and then gradually increasing towards the boundary over the length of the numerical beach [43].

$\mathbf{s}$ is initialised, using an analytical expression relating the value of $\mathbf{s}$ to the geometric coordinates of the NWT. Eqn. (4) is used in the current implementation which has been shown to produce good absorption [43]: 


$$
\begin{array}{r}
\operatorname{sand}(x)=-2 \cdot s_{\text {Max }}\left(\frac{\left(l_{\text {beach }}-x\right)}{l_{\text {beach }}}\right)^{3}+ \\
3 \cdot s_{\text {Max }}\left(\frac{\left(l_{\text {beach }}-x\right)}{l_{\text {beach }}}\right)^{2}
\end{array}
$$

where $l_{\text {beach }}$ is the length of the numerical beach, $x$ is the position within the numerical beach, equalling zero at the start and increasing to $l_{\text {beach }}$ at the NWT wall, and $s_{\text {Max }}$ stands for the maximum value of $\mathbf{s}$. In the simulations for this study, $l_{\text {beach }}$ and $s_{\text {Max }}$ are set to $6.75 \mathrm{~m}$ and $9 \mathrm{~Hz}$, respectively.

The $k-\omega S S T$ turbulence model is employed; however, based on the findings in [44], it is expected that the turbulence effects are minimal. Nevertheless, utilising the turbulence model has the beneficial effect of speeding up the simulations by almost $50 \%$, due to the damping of large amplitude, parasitic air velocities at the free surface, which would otherwise slow the simulation down because of the strict enforcement of the maximum allowable Courant number $(\mathrm{Co})$ employed by the adaptive time stepping scheme in OpenFOAM.

The spatial dimensions of the CNWT are based upon those of the physical test facility, as described in [3]: $9 \mathrm{~m} \times 12.8 \mathrm{~m} \times 6 \mathrm{~m}$ (WxLxH). A symmetry plane bisects the domain to reduce the computational overhead (cf. Fig. 5).

For the WEC structure, only the float is specifically represented in the numerical domain. Mass and inertial effects of the arm are indirectly captured in the numerical setup by using the CoM, mass and inertia of the complete physical system (float and arm).

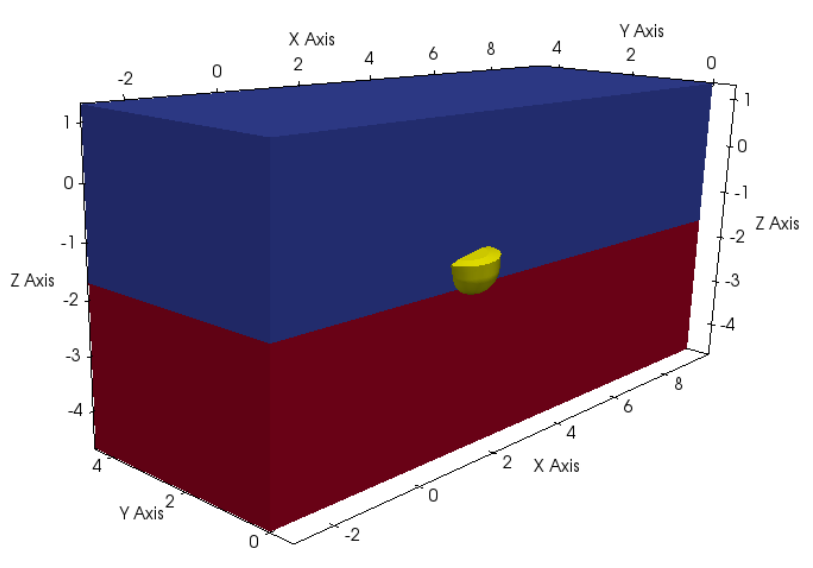

FIGURE 5: The CNWT domain, with the air depicted in blue, water in red and device (positioned out of its equilibrium) in yellow. A symmetry XZ plane bisects the tank.

\subsection{Solver Settings}

Due to the fundamental differences between the two DyMMMs, the optimal solver settings may differ for each. Therefore, to allow a fair comparison, the optimum solver settings for each method are first determined independently, and then each DyMMM is used with its own optimal solver settings in the later comparison. Note that the following tests to determine the solver setting are performed in a two-dimensional domain, due to the significant computational overhead for three dimensional tests.

4.1.1 Mesh Morphing For all simulations, adjustable time stepping with $C o$ control is employed. To determine the influence of the maximum allowable $C o$, both globally, $\mathrm{Co}_{\max }$, and for the phase fields, $C o_{\max , p f}$, simulations with different $\mathrm{Co}$ were performed. Based on the authors experience, the tested pairs of $\mathrm{Co}_{\max }$ and $\mathrm{Co}_{\max , p f}$ were $(0.30 .3)$, (0.8 0.3), (0.5 0.5), $(0.80 .5)$. Comparing the float heave position for the different cases, virtually no difference was found. However, run-time is decreased considerably, by a factor of 0.6 , between the test pairs $(0.30 .3)$ and $(0.80 .5)$. Hence, 0.8 and 0.5 were chosen for $C o_{\max }$ and $\mathrm{Co}_{\max , p f}$, respectively.

For the pressure-velocity coupling, the so-called PIMPLE algorithm is implemented in OpenFOAM. This algorithm blends the semi-implicit method for pressure linked equations (SIMPLE) and the pressure-implicit split-operator (PISO) algorithm. The blending algorithm is controlled by inner and outer iterations. Inner here refers to pressure-correction in a SIMPLE approach, whereas outer iterations refer to pressure-correction in the PISO approach [6]. To reduce the computational effort, the minimum number of inner and outer iterations needs to be found. For the case in hand, converged results are found for 2 inner and outer iterations.

As stated in Sec. 2.1, a distance-based diffusivity for the $\mathrm{MM}$ is implemented in OpenFOAM. Diffusivity is controlled through the setting of the inner and outer distance to the moving boundary. The dependency on these settings has been tested based upon [13], using inner distances and outer distances between $0.05-0.5 \mathrm{~m}$ and $2-3 \mathrm{~m}$, respectively. The results converge for an inner distance of $0.05 \mathrm{~m}$ and show negligible deviations of $<0.5 \%$ between the outer distance of $2 \mathrm{~m}$ and $3 \mathrm{~m}$. Finally, an inner distance of $0.05 \mathrm{~m}$ and an outer distance of $3 \mathrm{~m}$ are chosen. Parametrised by the float diameter $d_{\text {Float }}$, this represents approx. $d_{\text {Float }} / 20$ for the inner and $3 d_{\text {Float }}$ for the outer distance.

All other numerical solver settings have been chosen based on the appropriate tutorial cases provided in [1].

4.1.2 Overset Grids Similarly to MM, the PIMPLE algorithm is employed in the simulation using the OSG. Again, the minimum number of inner and outer iterations needs to be determined. Converged results are found for 2 inner and outer iterations. 
Bearing the solution method of the OSG in mind, the accuracy and efficiency of the interpolation schemes for the grid-togrid interpolation is of importance. In the current OpenFOAM implementation [1], three different interpolation schemes are available: inverseDistance (ID), tracking inverseDistance (tID) and cellVolumeWeight (CVW). For the given setup, CVW proved to be unavailable, so that only ID and tID could be tested. In the ID scheme, the influence of the donor cells on the interpolation is weighted based on the distance to the fringe cell. Donor cells closer to the fringe cell have a larger influence on the interpolation, whereas donor cells further way from the fringe cell have less influence [45]. Unfortunately, detailed information on the differences between the ID and IID is not available. Testing the two interpolation schemes, no differences in the results was found. However, the tID required longer run times, therefore the ID scheme is selected for subsequent use.

All other numerical solver settings have been chosen based on the appropriate tutorial cases in [1].

\subsection{Mesh Settings}

4.2.1 Mesh Morphing From the physical setup of the WEC, a xz plane symmetry can be identified. This property is exploited in the numerical setup as shown in Fig. 5. To prove the validity of this assumption, numerical simulations have been performed in a fully three-dimensional (3D) domain and a 3D domain including a symmetry plane. Results are shown in Fig. 6 and highlight the validity of the symmetry conditions.

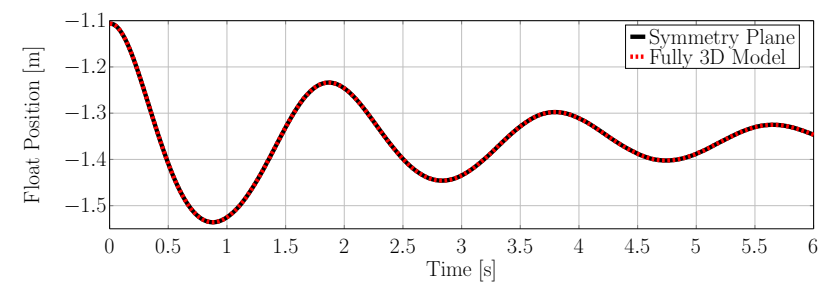

FIGURE 6: Results from numerical simulations performed in fully $3 \mathrm{D}$ domain and in a 3D domain including a symmetry $\mathrm{XZ}$ plane

The layout of the numerical grid, including the boundary conditions (BCs), is depicted in Fig. 7. Mesh refinement is employed in the interface region, resulting in cubical cells with an edge size of $0.03 \mathrm{~m}$. Around the body, an additional refinement layer is used, resulting in an edge size of $0.015 \mathrm{~m} \approx 70$ cells per $d_{\text {Float }}$. Cell stretching is applied towards the boundaries to provide additional damping and to reduce the total number of cells. The total cell count of the domain adds up to $1.2 \mathrm{M}$ cells. Cell sizes have been determined through a grid convergence study. Each MM test is run with a parallel simulation using 22 processors on a single computing node, with the domain decomposed using the Scotch method implemented in OpenFOAM [46].

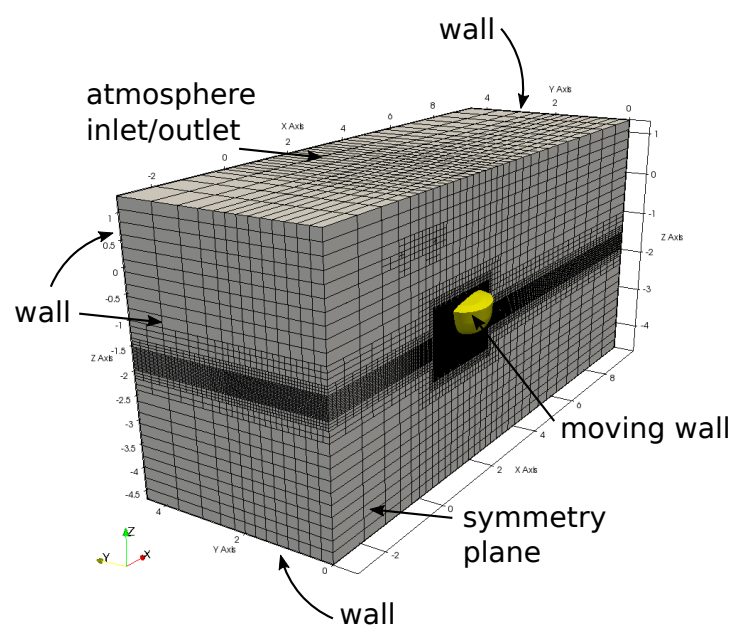

FIGURE 7: Mesh layout for the MM simulations. Mesh refinement is employed around the free surface interface and the body. Cell stretching is used towards the far field boundary

4.2.2 Overset Grids For the OSG, the MM grid setting is used as a basis, with slight modifications applied. Mesh refinement towards the free surface, as well as cell stretching, is employed (cf. Fig. 8). Also, grid cell sizes in the interface region and around the body are taken from the MM setting. However, preliminary studies revealed that the application of a symmetry plane, as in the MM case, is not yet supported for OSG. Hence, simulations have to be performed in a fully 3D domain.

Furthermore, a number of geometric issues must be considered when setting up the OSG domain. One is the influence of the size of the body-fitted grid. For the analysis, the body-fitted grid size is parametrised by $T_{r}=R_{o} / d_{\text {Float }}$, where $R_{o}$ is the extension of the body-fitted grid in the x direction (cf. Fig. 9). In the 2D domain, simulations for $T_{r}=1.4, T_{r}=1.6, T_{r}=1.8$ and $T_{r}=2.2$ were tested. Negligible differences of $<0.5 \%$ were found in the float position and total volume fraction of the liquid phase. Finally, a rather conservative setting of $T_{r}=2.2$ has been chosen to comply with the MM settings. The grid layout is depicted in Figs. 9 and 10.

Significant influence on the occurrence of interpolation errors is also found for differing cell sizes between the body-fitted and background grids in the overlayed region (marked in dashed red in Fig. 10). Cell sizes in the two regions must be of similar size to avoid such interpolation errors. This, in turn, determines the size of the overlayed region in the background grid. The overlayed region should be big enough so that the body-fitted grid always falls within the region throughout the simulation.

The complete CNWT layout, including the BCs for the background and body-fitted grids, is depicted in Fig. 10. The 
background grid is marked in blue, the body-fitted grid in red and the WaveStar structure is marked in yellow. The total cell count of the domain adds up to $6.6 \mathrm{M}$ cells.

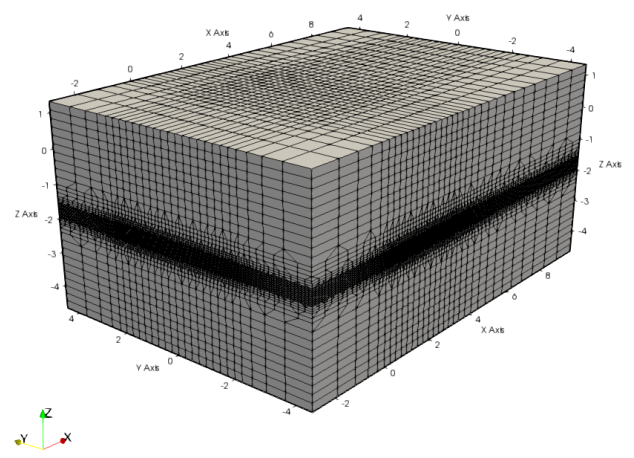

FIGURE 8: Mesh layout of the background mesh for OSG simulations. Due to missing support for symmetry planes, a fully 3D mesh is used

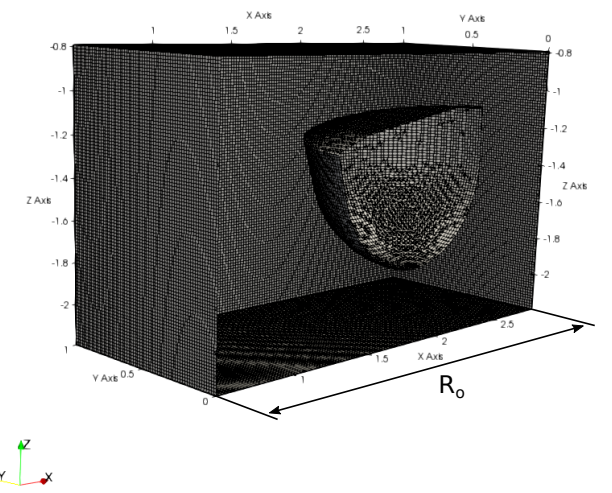

FIGURE 9: One half of the mesh layout of the body-fitted grid for OSG simulations. For clarity, only the mesh on the outer and body patches are shown

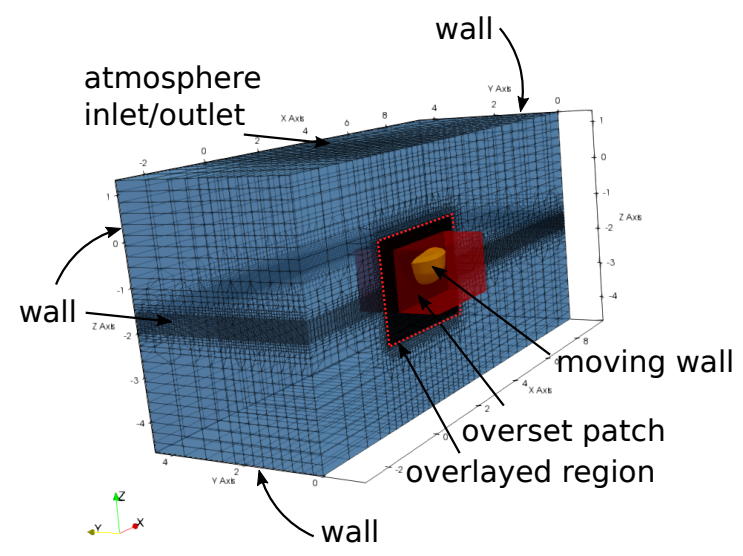

FIGURE 10: Mesh setup for OSG simulations, showing the background grid (blue), body-fitted grid (red) and WEC body (yellow)

\section{RESULTS}

This section presents the results for two different DyMMMs and compares body motion to the experimental tank test data.

\subsection{Mesh Morphing}

The numerical simulations are validated against experimental tank test data. Fig. 11 shows the time trace of the float position in the heave DOF for test case FD1. Overall, very good agreement between experimental and numerical results with a mean error of $<0.5 \%$ can be observed. At the upstroke peaks, at $1.8 \mathrm{~s}$ and $3.7 \mathrm{~s}$, a slight overshoot of the numerical solution can be seen, possibly due to parasitic friction effects in the experiments. At the downstroke troughs, at $0.9 \mathrm{~s}$ and $2.8 \mathrm{~s}$, the two results coincide. Furthermore, the natural period of the oscillation is captured accurately.

As shown in Fig. 12, very good agreement between the experimental and numerical results with a mean error of $0.5 \%$ is also achieved for test case FD2. Slightly larger deviations at the up- and downstroke peak displacements can be observed. The larger deviations might be explained due to the larger relative influence of the friction effects for the smaller amplitude oscillations in the FD2 test. Again, the natural frequency of the system is well captured.

For the validation study shown, it can be stated that the MM does deliver physically correct results and is able to reproduce the experimental tests with sufficient accuracy.

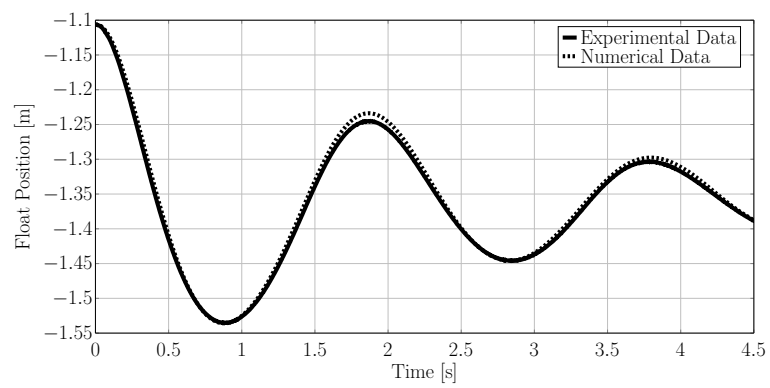

FIGURE 11: Numerical and experimental results for FD1

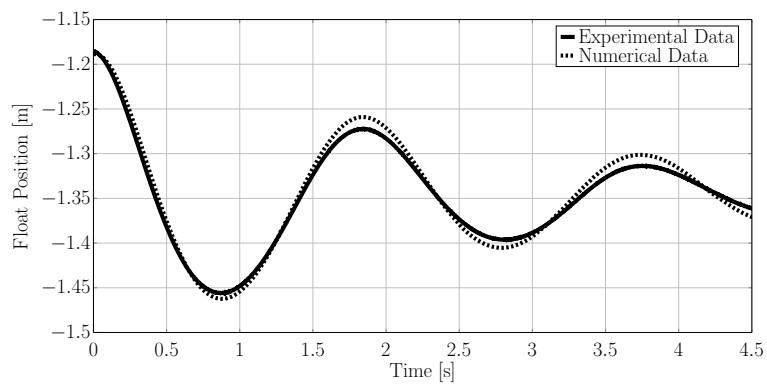

FIGURE 12: Numerical and experimental results for FD2 


\subsection{Overset Grids}

Due to the extensive computational overhead (cf. Tab. 2), the simulated time for OSG is limited to fractions of the time shown in Figs. 11 and 12. Furthermore, only a single test case, FD1, is considered for the validation of the OSG.

Fig. 13 shows the time trace of the heave float position from four different simulations for the FD1 test: Experimental benchmark data (solid black line), OSG on 22 cores using the scotch decomposition (dashed blue), OSG on 18 cores using the simple decomposition (dashed green line) and OSG on a single core (dashed red line).

Initially, OSG simulations were performed on 22 cores using the Scotch decomposition. This decomposition method has been used for all MM simulations and splits the domain with the goal of reducing the number of subdomain boundaries. In Fig. 13 , it can clearly be seen that the results from this simulation differ from the benchmark case. Since the Scotch decomposition automatically splits the domain into the user-defined number of subdomains, it is likely that the overset patch is split onto different cores, which may in turn, lead to additional errors through more implicit interpolation [1] or more severe violation of the mass conservation.

Therefore, simulations on a single core were performed, to identify any possible influence of the decomposition method on the results. As shown in Fig. 13, by not utilising any decomposition with the OSG, overall agreement with the benchmark was achieved, with comparable accuracy as the MM method. The mean deviation between experimental and numerical data is $0.6 \%$. However, a shift in the natural period of the oscillating system can be observed. This potentially increases the deviation over a longer simulation time.

In a subsequent simulation, the OSG domain has been decomposed using the simple decomposition method implemented in OpenFOAM. Here, the user can manually set the number of subdomains in $\mathrm{x}-, \mathrm{y}-$ and $\mathrm{z}$-direction. The layout of the subdomains for the current case is illustrated in Fig. 14. Using this method, it was possible to split the domain into a total of 18 subdomains while avoiding the division of the overset patch. The results in Fig. 13 reveal agreement between the single and multicore simulations up to $0.2 \mathrm{~s}$. However, for later time instances, large deviations between experimental data and OSG multi core simulations can be observed, suggesting, that domain decomposition generally introduces numerical errors, not only when the overset patch is split. This is found to be a huge drawback of the OSG method in its current implementation and needs further investigation to determine the source of the additional errors.

From the results in Figs. 11 and 13, it can be stated that OSG on a single core and MM perform with similar accuracy. To compare the efficiency of the two methods, run times are assessed in Tab. 2. The run time in seconds $\left(s_{r t}\right)$ is normalised by the simulated time in seconds $\left(s_{\text {sim }}\right.$.). The $2 \mathrm{D}$ case in Tab. 2 refers to the setup used for the assessment of optimal solver settings in Sec.
4.1. The $3 \mathrm{D}$ cases refer to the setup used to generate the results shown in Fig. 13.

It can clearly be seen that the MM simulations require less time per simulated second. For the $2 \mathrm{D}$ case, an increase by a factor of 3.7 was found. For the 3D cases, the increase is even greater with factors more than 10 and 57 for the single and multicore cases, respectively. Comparing the 3D multi core cases, the different total number of cores has to be taken into account, making the comparison harder.

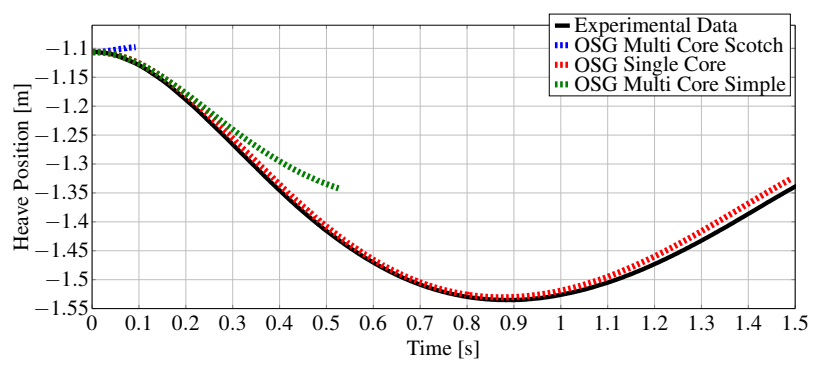

FIGURE 13: Numerical results of the heave float position for OSG on a single core, OSG on multiple cores using the scotch and simple decomposition method
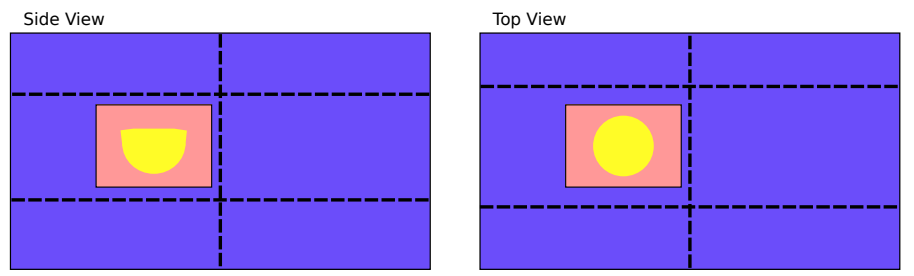

FIGURE 14: Schematic of the subdomain layout for OSG simulations using the simple decomposition method

TABLE 2: NORMALISED RUNTIME FOR MESH MORPHING AND OVERSET GRIDS

\begin{tabular}{llll}
\hline & \multirow{2}{*}{ 2D Case } & \multicolumn{2}{c}{ 3D Case } \\
& & Sinlge Core & Multi Core \\
\hline MM & $172 s_{r t} / s_{\text {sim. }}$ & $58 \mathrm{k} s_{r t} / s_{\text {sim. }}$ & $10 \mathrm{k} s_{r t} / s_{\text {sim. }}$ (22 Cores) \\
OSG & $632 s_{r t} / s_{\text {sim. }}$. & $614 \mathrm{k} s_{r t} / s_{\text {sim. }}$ & $570 \mathrm{k} s_{r t} / s_{\text {sim. }}$ (18 Cores) \\
\hline
\end{tabular}

$s_{r t}=$ seconds run time; $s_{\text {sim. }}=$ seconds simulated time 


\section{Conclusion}

In this paper, a comparison study between the OSG and MM is undertaken by comparing numerical results with experimental tank tests data from free decay test of the WaveStar device. From the results, the following conclusions can be drawn:

i) Only without parallelization, OSG and MM reach comparable accuracy

ii) OSG requires an extremely careful model setup

iii) OSG increases the computational overhead

iv) For simulations, in which MM would lead to numerical instability and computational efficiency is not crucial, OSG can be a viable option

In the OpenFOAM fork [1], the considered OSG algorithm is still in an early development stage. Hence, further improvements in later releases are likely and should be considered in a future assessment of the method.

\section{ACKNOWLEDGMENT}

This paper is based upon work supported by Science Foundation Ireland under Grant No. 13/IA/1886. Furthermore, the authors would like to acknowledge Morten Jakobsen and Morten Kramer of Aalborg University for providing the experimental tank test data and Ed Ransley of Plymouth University for his advice on the numerical setup.

\section{REFERENCES}

[1] ESI, 2017. OpenFOAM v1706 Release Notes. https://openfoam.com/releases/openfoamv1706/numerics.php.

[2] FSB, 2017. foam-extend Release Notes. http://foamextend.fsb.hr/blog/2017/01/17/release-of-overset-mesh-infoam-extend/.

[3] Jakobsen, M., Beatty, S., Iglesias, G., and Kramer, M., 2014. "Characterization of loads on a hemispherical point absorber wave energy converter". International Journal of Marine Energy.

[4] de O. Falcao, A. F., 2010. "Wave energy utilization: A review of technologies". Renewable and Sustainable Energy Reviews.

[5] Cruz, J., ed., 2008. Ocean Wave Energy. Springer.

[6] Windt, C., Davidson, J., and Ringwood, J., 2017. "High-fidelity Numerical Modelling of Ocean Wave Energy Systems: a Review of Computational Fluid Dynamics-based Numerical Wave Tanks". submitted to Renewable and Sustainable Energy Reviews.

[7] Folley, M., ed., 2016. Numerical Modelling of Wave Energy Converters. Academic Press.

[8] Giorgi, G., and Ringwood, J. V., 2016. "Implementation of latching control in a numerical wave tank with regular waves". Journal of Ocean Engineering and Marine Energy, 2(2), apr, pp. 211-226.

[9] Causon, D., Ingram, D., Mingham, C., Yang, G., and Pearson, R., 2000. "Calculation of shallow water flows using a cartesian cut cell approach". Advances in Water Resources, $\underline{\mathbf{2 3}}(5)$, feb, pp. 545-562.

[10] Ingram, D., Causon, D., and Mingham, C., 2003. "Developments in cartesian cut cell methods". Mathematics and Computers in Simulation, 61(3-6), jan, pp. 561-572.

[11] Yu, Y.-H., and Li, Y., 2013. "Reynolds-averaged navier-stokes simulation of the heave performance of a two-body floating-point absorber wave energy system". Computers \& Fluids, 73, mar, pp. 104-114.

[12] Palm, J., Eskilsson, C., Paredes, G. M., and Bergdahl, L., 2016. "Coupled mooring analysis for floating wave energy converters using CFD: Formulation and validation". International Journal of Marine Energy, 16, dec, pp. 8399.

[13] Ransley, E., Greaves, D., Raby, A., Simmonds, D., Jakobsen, M., and Kramer, M., 2017. "RANS-VOF modelling of the wavestar point absorber". Renewable Energy, 109, aug, pp. 49-65.

[14] Davidson, J., Windt, C., Giorgi, G., Genest, R., and Ringwood, J., 2018. "Chapter: Evaluation of energy maximising control systems for wave energy converters using OpenFOAM". OpenFOAM - Selected papers from the 11th workshop.

[15] Liu, Z., Hyun, B.-S., and Hong, K.-Y., 2008. "Application of numerical wave tank to owc air chamber for wave energy conversion". In Proceedings of the Eighteenth International Offshore and Polar Engineering Conference.

[16] Jin, J., Liu, Z., Hong, K.-Y., and Hyun, B.-S., 2012. “3d numerical investigation on reservoir system for an overtopping wave energy convertor". Journal of Navigation and Port Research, 36(2), mar, pp. 97-103.

[17] Dolguntseva, I., Chen, W., Savin, A., Zhang, Y., Li, W., Svensson, O., and Leijon, M., 2017. "Numerical modelling of a point-absorbing wave energy converter in irregular and extreme waves". Applied Ocean Research, 63, pp. $90-$ 105.

[18] Schmitt, P., Bourdier, S., Sarkar, D., Renzi, E., Dias, F., Doherty, K., Whittaker, T., and van 't Hoff, J., 2012. "Hydrodynamic loading on a bottom hinged oscillating wave surge converter". In Proceedings of the Twenty-second (2012) International Ocean and Polar Engineering Conference.

[19] Schmitt, P., and Elsässer, B., 2017. "The application of froude scaling to model tests of oscillating wave surge converters". Ocean Engineering.

[20] Sharma, N., and Patankar, N. A., 2005. "A fast computation technique for the direct numerical simulation of rigid particulate flows". Journal of Computational Physics, 205(2), may, pp. 439-457. 
[21] Flow Science. Flow-3D Favor. https: //www.flow3d.com/resources/ cfd-101/modeling-techniques/ no-loss-with-favor/ Lasy accessed 12/05/2017.

[22] Benek, J., Steger, J., and Dougherty, F., 1983. "A flexible grid embedding technique with application to the euler equations". In 6th Computational Fluid Dynamics Conference Danvers, American Institute of Aeronautics and Astronautics.

[23] Ahmad, J., and Duque, E. P. N., 1996. "Helicopter rotor blade computation in unsteady flows using moving overset grids". Journal of Aircraft.

[24] Prewitt, N. C., Belk, D. M., and Shyy, W., 2000. "Parallel computating of overset grids for aerodynamic problems with moving objects". Progress in Aerospace Sciences.

[25] Li, Y., Paik, K., Xing, T., and Carrica, P., 2012. "Dynamic overset cfd simulations of wind turbine aerodynamics". Renewable Energy.

[26] Boger, D., and Dreyer, J., 2006. "Prediction of hydrodynamic forces and moments for underwater vehicles using overset grids". In Proceedings of the 44TH AIAA AEROSPACE SCIENCES MEETING AND EXHIBIT.

[27] Mofidi, A., and Carrica, P., 2014. "Simulations of zizag maneuvers for a container ship with direct moving rudder and propeller". Computers \& Fluids.

[28] Shen, Z., Wan, D., and Carrica, P., 2015. "Dynamic overset grids in openfoam with application to kcs self-propulsion and maneuvering". Ocean Engineering.

[29] Stansby, P., Gu, H., Moreno, E. C., and Stallard, T., 2015. "Drag minimisation for high capture width with three float wave energy converter m4". In Proceedings of the 11th European Wave and Tidal Energy Conference.

[30] Bharath, A., Penesis, I., Nader, J.-R., and Macfarlane, G., 2016. "Non-Linear CFD Modelling of a Submerged Sphere Wave Energy Converter". In Proceedings of the 3rd Asian Wave and Tidal Energy Conference.

[31] Coiro, D. P., Troise, G., Calise, G., and Bizzarrini, N., 2016. "Wave energy conversion through a point pivoted absorber: Numerical and experimental tests on a scaled model". Renewable Energy, 87, mar, pp. 317-325.

[32] Lu, X., Denver, D., Chandar, J., Chen, Y., and Lou, J., 2017. "An overlapping domain decomposition based nearfar field coupling method for wave structure interaction simulations". Coastal Engineering, pp. 37-50.

[33] Elhanafi, A., Macfarlane, G., Fleming, A., and Leong, Z., 2017. "Intact and damage survivability of an offshore floating-moored OWC device". In Proceedings of the Twenty-seventh (2017) International Ocean and Polar Engineering Conference.

[34] Elhanafi, A., Macfarlane, G., Fleming, A., and Leong, Z., 2017. "Experimental and numerical investigations on the hydrodynamic performance of a floating-moored oscillat- ing water column wave energy converter". Applied Energy.

[35] Elhanafi, A., Macfarlane, G., Fleming, A., and Leong, Z., 2017. "Experimental and numerical investigations on the intact and damage survivability of a floating-moored oscillating water column device". Applied Ocean Research.

[36] Jung, M., and Kwon, O., 2006. "A parallel unstrutured overset mesh technique for unsteady flow simulations". In Proceedings of the 4th International Conference on Computational Fluid Dynamics ICCFD.

[37] Ferziger, J. H., and Peric, M., 2001. Computational Methods for Fluid Dynamics. Springer-Verlag GmbH.

[38] Jasak, H., and Rusche, H., 2010. Dynamic mesh handling in OpenFOAM. Presentation at the 2010 OpenFOAM Workshop.

[39] Thompson, J., 1999. Handbook of Grid Generation. CRC Press LLC.

[40] Celeritas Simulation Technology, LLC, 2011. Overset grid assembly process. http://celeritassimtech. $\mathrm{com} /$ ?page_id=113.

[41] Wang, Z., and Parthasarathy, V., 2000. "A fully automated Chimera methodology for multiple moving body problems". International Journal for Numerical methods in Fluids.

[42] Weller, H. G., Tabor, G., Jasak, H., and Fureby, C., 1998. "A tensorial approach to computational continuum mechanics using object-oriented techniques". Computers in Physic, 12(6), pp. $620-631$.

[43] Schmitt, P., Windt, C., Davidson, J., Ringwood, J., and Whittaker, T., 2017. "The efficient application of an impulse source wave maker to cfd simulations". submitted to Coastal Engineering.

[44] Windt, C., Davidson, J., and Ringwood, J., 2017. Turbulence modelling for ocean wave energy systems. Presentation at the 12th OpenFOAM Workshop, Exeter. https:

//www.researchgate. net/publication/ 319448474_Turbulence_Modelling_for_ Ocean_Wave_Energy_Systems.

[45] ArcGIS, 2017. How inverse distance weighted (idw) interpolation works. http://webhelp.esri.com/ arcgisdesktop $/ 9.2 /$ index. $\mathrm{cfm}$ ? TopicName= How_Inverse_Distance_Weighted_(IDW) _interpolation_works.

[46] CFD Direct, 2017. OpenFOAM v5 user guide: 3.4 running applications in parallel. https: //cfd.direct/openfoam/user-guide/ running-applications-parallel/. 